\title{
锂离子电池正极材料 $\mathrm{LiNi}_{0.8} \mathrm{Co}_{0.15} \mathrm{Al}_{0.05} \mathrm{O}_{2}$ 表面包覆的 研究进展
}

\author{
丛亮, 雷凯翔, 王纪伟, 王建斌, 孟焕菊, 程方益*, 陈军 \\ 南开大学化学学院, 先进能源材料化学教育部重点实验室, 天津 300071 \\ *联系人, E-mail: fycheng@ nankai. edu. cn
}

2016-03-10 收稿, 2016-04-06 修回, 2016-04-11 接受, 2016-05-09 网络版发表 国家自然科学基金 $(21231005,21322101)$ 、教育部新世纪优秀人才支持计划(ACET-13-0296)和重点科技项目(113016A)资助

摘要高比能、长寿命锂离子电池正极材料是目前电池研发热点. 元素组成为 $\mathrm{LiNi}_{0.8} \mathrm{Co}_{0.15} \mathrm{Al}_{0.05} \mathrm{O}_{2}(\mathrm{NCA})$ 的高镍 正极材料相比于传统的层状 $\mathrm{LiCoO}_{2}$ 具有价格便宜和比容量高的优点, 已逐渐进入商品化应用阶段. 该材料仍存在 不可逆容量损失大、高温性能差等问题，表面包覆是提升材料性能的有效途径. 本文总结了NCA材料表面包覆的 研究进展，并对其未来的研究方向进行了展望.

关键词锂离子电池，正极材料，金属氧化物，性能优化，表面包覆

随着信息化社会发展和能源产业结构调整，电 池在便携式电子设备、电动交通工具以及可再生能 源储能等领域中的应用持续增长. 锂离子电池相对 于其他电池体系具有能量密度高、循环性能好、无记 忆效应等突出优点, 得到迅猛发展 ${ }^{[1 \sim 3]}$. 锂离子电池 的性能主要取决于参与电极反应的活性物质, 负极 的发展速度要快于正极 ${ }^{[4]}$, 如何进一步提升正极材料 的性能是目前研发的热点和重点.

具有层状结构的 $\mathrm{LiCoO}_{2}$ 是当前主要的商品化锂 离子电池正极材料, 其综合性能优异, 但成本较高、Co 存在毒性, 制约了其更大规模应用. $\mathrm{LiNiO}_{2}$ 晶体结构 类似, 成本较低且更加环保, 但结构稳定性较差. 通 过用其他元素(如 $\mathrm{Co}^{[6,7]}, \mathrm{Al}^{[8,9]}, \mathrm{Mn}^{[10,11]}$ 等)部分取代 $\mathrm{LiNiO}_{2}$ 中的 $\mathrm{Ni}^{3+}$, 可以改善高容量电极材料 $\mathrm{LiNiO}_{2}$ 的 结构稳定性, 减少循环过程中的不可逆容量损失. 其 中, 掺杂 $\mathrm{Co}, \mathrm{Al}$ 两种元素而获得的 $\mathrm{LiNi}_{1-x-y} \mathrm{Co}_{x} \mathrm{Al}_{y} \mathrm{O}_{2}$ 材料的电化学性能突出 ${ }^{[12 ~ 14]} . \mathrm{LiNi}_{1-x-y} \mathrm{Co}_{x} \mathrm{Al}_{y} \mathrm{O}_{2}$ 属于 $\alpha-\mathrm{NaFeO}_{2}$ 型晶体结构, $\mathrm{R}-3 \mathrm{~m}$ 空间点群, $\mathrm{Ni}, \mathrm{Co}, \mathrm{Al}$ 与 $\mathrm{O}$
通过八配位成键, 形成供 $\mathrm{Li}^{+}$脱嵌的二维层状通道, 如图 1(a)所示. 在具有不同 $\mathrm{Ni}: \mathrm{Co}: \mathrm{Al}$ 元素比例的系 列材料中, 对元素组成为 $\mathrm{LiNi}_{0.8} \mathrm{Co}_{0.15} \mathrm{Al}_{0.05} \mathrm{O}_{2}$ (NCA) 的材料研究最为充分, 已经逐渐实现商品化. NCA材 料的典型充放电曲线如图 1(b)所示. 其电压平台与 $\mathrm{LiCoO}_{2}$ 相似，但放电比容量可达 $200 \mathrm{mAh} / \mathrm{g}$, 较 $\mathrm{LiCoO}_{2}(\sim 140 \mathrm{mAh} / \mathrm{g})$ 有很大提高.

$\mathrm{NCA}$ 材料在使用过程中仍存在着一些问题，主 要有以下 2 点: (1) 充电过程中, 由于 $\mathrm{Ni}^{2+}$ 和 $\mathrm{Li}^{+}$的半径 非常接近，部分 $\mathrm{Ni}^{2+}$ 会占据 $\mathrm{Li}^{+}$的空位，发生离子混 排，造成材料的不可逆容量损失; (2) 材料中的 $\mathrm{Ni}$ 在 处于高氧化态时 $\left(\mathrm{Ni}^{3+}\right.$ 或 $\left.\mathrm{Ni}^{4+}\right)$ 具有很强的不稳定性, 高温下会导致材料结构发生改变 ${ }^{[15]}$, 并容易与电解 液发生副反应，造成容量衰减. 针对以上问题，可通 过掺杂 $\mathrm{Mg}, \mathrm{Mn}$ 等元素来合成 $\mathrm{LiNi}_{1-x-y-z} \mathrm{Co}_{x} \mathrm{Al}_{y} \mathrm{M}_{z} \mathrm{O}_{2}$ 四 元材料 ${ }^{[16,17]}$ 以及对三元材料进行表面包覆来对材料 的性能进行改善. 掺杂可以稳定材料的晶格结构, 降 低阳离子混排程度, 减少充放电过程中的不可逆容 

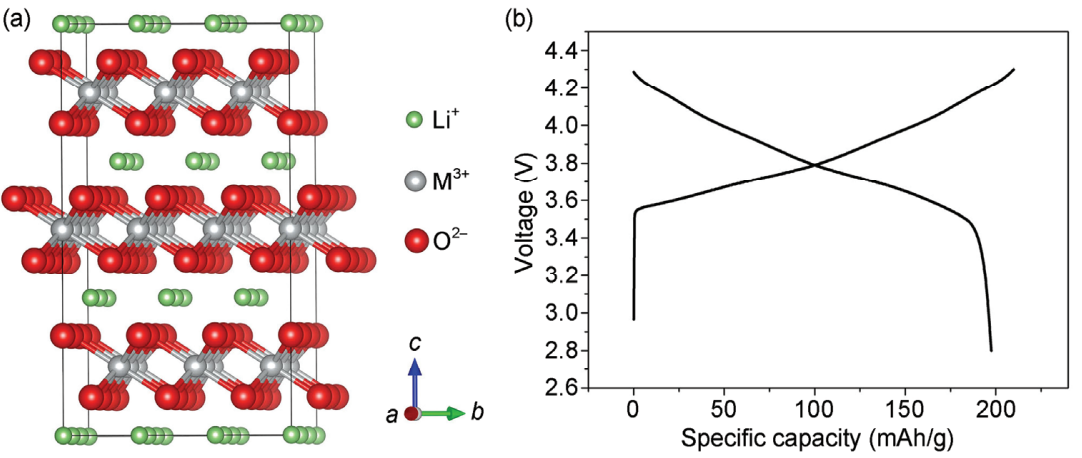

(c)
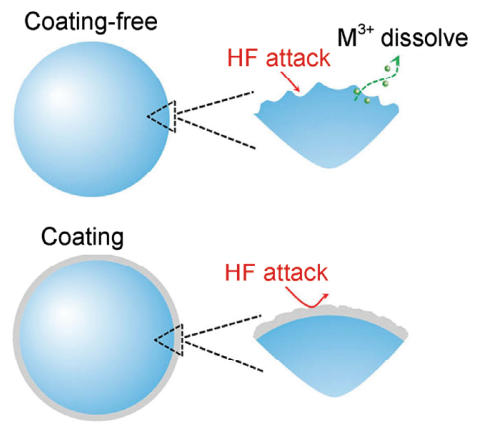

图 1 (网络版彩色)(a) $\mathrm{LiMO}_{2}(\mathrm{M}=\mathrm{Ni}, \mathrm{Co}, \mathrm{Al}$ )的晶体结构示意图; (b) $\mathrm{NCA}$ 的典型充放电曲线; (c) 包覆作用图示

Figure 1 (Color online) (a) Crystal structure of $\mathrm{LiMO}_{2}(\mathrm{M}=\mathrm{Ni}, \mathrm{Co}, \mathrm{Al})$. (b) Typical charge-discharge profile of NCA. (c) Mechanism of coating effect

量损失, 是从“材料内部”来提高性能. 而表面包覆则 可以降低电极材料与电解液的直接接触面积, 减少 电解液中的HF对材料的腐蚀作用, 从而抑制副反应 的发生(图 1(c) ) ${ }^{[18,19]}$, 是从“材料外部” 来解决问题. 相比于掺杂, 人们更多地采用表面包覆来对材料进 行改性 ${ }^{[20]}$.

目前用于NCA材料的包覆物质有碳材料、氧化 物、氟化物、锂化物、复合氧化物、磷酸盐和聚合物 等. 本文按照表面包覆物的种类, 对不同种类包覆物 的包覆方法和包覆作用的研究进展进行综述.

\section{1 碳材料}

在正极材料常用的诸多包覆物中, 碳材料因为 具有较高的导电性, 可以提升正极材料的循环和倍 率性能. 考虑到通常需要通过有机物在高温下热解 来实现碳包覆, 而高温下三元正极材料容易被生成 的碳还原, 所以关于碳材料对三元正极材料进行包 覆的报道相对较少. Chung等人 ${ }^{[21]}$ 采用十二烷基硫酸 钠做碳源, 将 $\mathrm{NCA}$ 材料与十二烷基硫酸钠混合均匀
后在空气中 $600^{\circ} \mathrm{C}$ 煅烧 $5 \mathrm{~h}$ 得到碳包覆的 $\mathrm{NCA} / \mathrm{C}$ 材料, 如图2(a), (b)所示. 在2.8 4.3 V 的电压区间, 0.1, 0.5, 1 和 3 C 倍率下进行充放电, $\mathrm{NCA} / \mathrm{C}$ 的放电比容量分别 为 $183,165,140$ 和 $83 \mathrm{mAh} / \mathrm{g}$, 相比于未包覆材料的 $181,160,128$ 和 $46 \mathrm{mAh} / \mathrm{g}$, 在大倍率条件下有较大提 高. 同时材料的循环性能也得到了改善. NCA/C 在 $0.1 \mathrm{C}$ 倍率下循环 40 圈的容量保持率为 $93 \%$, 高于未 包覆材料的 $86 \%$ (图2(c)).

Yoon等人 ${ }^{[22]}$ 采用高能机械球磨法, 将 NCA与石 墨烯在氞气保护下 $200 \mathrm{r} / \mathrm{min}$ 球磨 $30 \mathrm{~min}$, 得到 NCA石墨烯复合材料. 在 $55.6 \mathrm{~mA} / \mathrm{g}$ 的电流下循环 80 次, $\mathrm{NCA}$-石墨烯复合材料的首次放电比容量为 $180 \mathrm{mAh} / \mathrm{g}$, 容量保持率为 $97 \%$, 而未包覆材料的首次放电比容 量为 $172 \mathrm{mAh} / \mathrm{g}$, 容量保持率为 $91 \%$. 所包覆的石墨 烯增强了材料的导电性, 从而降低了电池的极化. 相 比于其他碳包覆实验，该方法采用石墨烯进行包覆， 不需高温煅烧而直接获得碳源, 更加节能环保, 但还 需要考虑添加石墨烯带来的成本增加并提高石墨烯 包覆层的均匀程度.
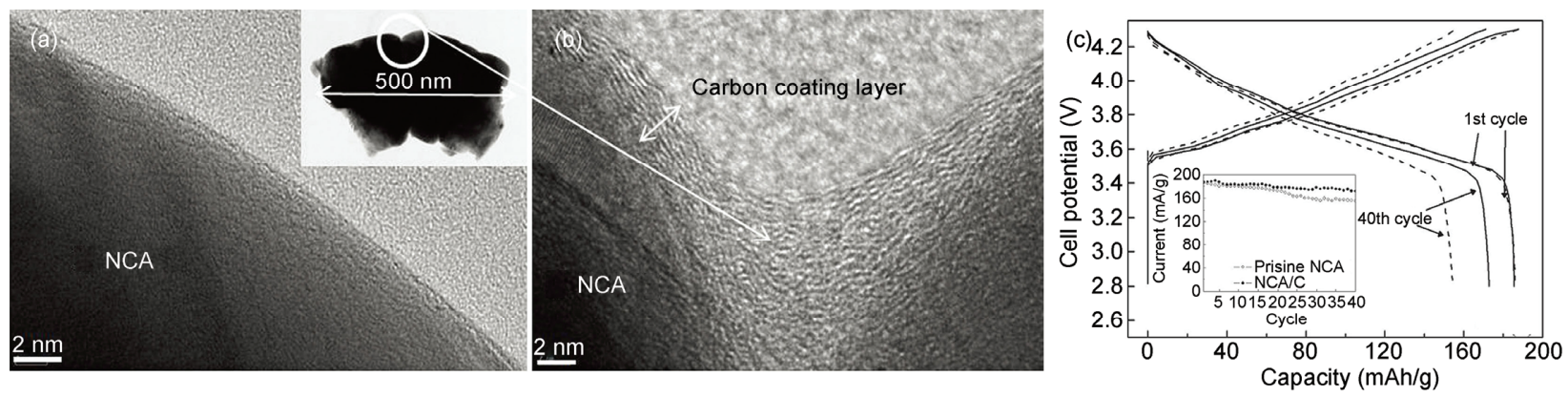

图 $2 \mathrm{NCA}(\mathrm{a})$ 和NCA/C (b)的场发射透射电子显微镜(FE-TEM)图像及循环性能对比(c) ${ }^{[21]}$

Figure 2 FE-TEM images of NCA (a) and NCA/C (b). (c) Cycling performances ${ }^{[21]}$ 


\section{2 氧化物}

在对 NCA材料的表面包覆改性中, 氧化物被广 泛采用. Cho等人 ${ }^{[23]}$ 用纳米 $\mathrm{SiO}_{2}(\sim 100 \mathrm{~nm})$ 对 NCA材料 进行包覆, 混合 $2 \mathrm{~h}$ 后在空气中 $700^{\circ} \mathrm{C}$ 炦烧 $5 \mathrm{~h}$, 得到 $\mathrm{NCA} / \mathrm{SiO}_{2}$ 材料. 在 $60^{\circ} \mathrm{C}$ 条件下以 $1 \mathrm{C}$ 倍率进行测试, 循环 40 次后, 未包覆材料的比容量为 $41 \mathrm{mAh} / \mathrm{g}$, 而包 覆后材料的比容量为 $101 \mathrm{mAh} / \mathrm{g}$, 包覆效果十分明显. $\mathrm{SiO}_{2}$ 分布在材料的表面, 减少了 $\mathrm{Ni}^{2+}$ 在电极与电解液 界面的形成.

通过溶胶凝胶法可以在 NCA 材料表面包覆 $\mathrm{CeO}_{2}{ }^{[24]}$. 电化学测试表明, $0.02 \%$ (物质的量比)包覆 量的材料具有最佳性能. 在 $0.5 \mathrm{C}, 2.75 \sim 4.3 \mathrm{~V}$ 下循环 100 次, $\mathrm{NCA} / \mathrm{CeO}_{2}$ 材料的容量保持率为 $85.96 \%$, 而未 包覆材料的容量保持率只有 $78.22 \%$. 包覆的 $\mathrm{CeO}_{2}$ 在 活性材料和电解液之间起到隔离作用, 抑制了材料 与电解液的副反应. 而具有强氧化性的 $\mathrm{CeO}_{2}$ 也会与 电解液中的痕量HF反应, 从而减少了电解液对活性 材料的侵蚀.

$\mathrm{TiO}_{2}$ 也可以作为包覆物, 通过钛酸四丁酯水解
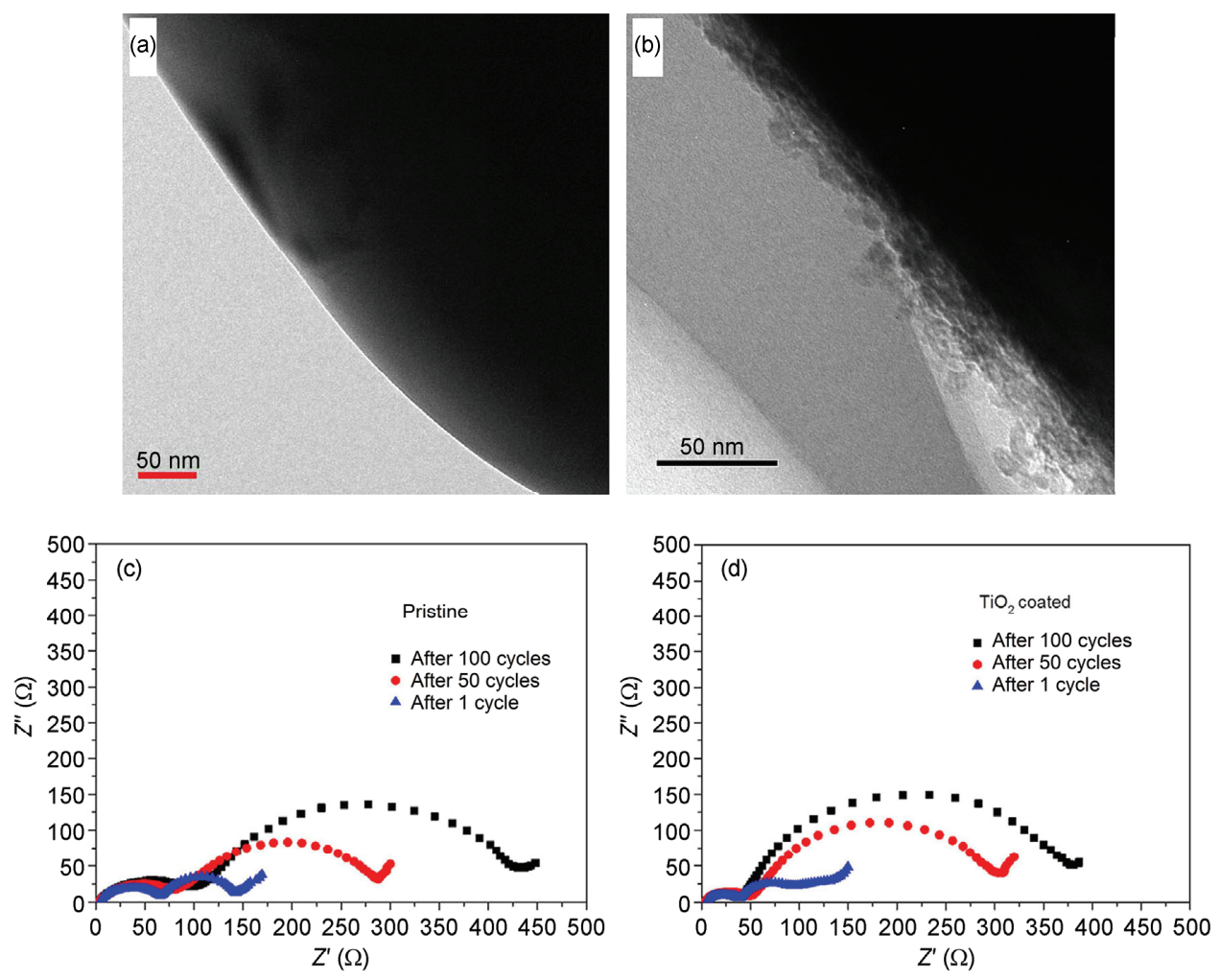

图 3 (网络版彩色) NCA(a)和 $\mathrm{NCA} / \mathrm{TiO}_{2}$ (b)的TEM图像; $\mathrm{NCA}(\mathrm{c})$ 和 $\mathrm{NCA} / \mathrm{TiO}_{2}(\mathrm{~d})$ 的阻抗图 ${ }^{[25]}$

Figure 3 (Color online) TEM images of NCA (a) and $\mathrm{NCA} / \mathrm{TiO}_{2}$ (b). EIS plot of NCA (c) and $\mathrm{NCA} / \mathrm{TiO}_{2}(\mathrm{~d})^{[25]}$ 
生成HF, 对电极材料造成腐蚀. 由于氟化物对 HF稳 定, 可以有效地防止HF对电极材料的侵蚀, 因此也可 采用氟化物对电极材料进行包覆. Lee等人 ${ }^{[27]}$ 将自制

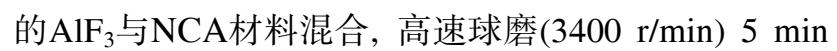
后获得 $\mathrm{NCA} / \mathrm{AlF}_{3}$ 材料, 包覆层大约为 $50 \mathrm{~nm}$. 包覆 后, 材料的循环性能、倍率性能和热稳定性均有了一 定提高, 尤其是高温时的循环性能. $55^{\circ} \mathrm{C}, 1 \mathrm{C}$ 倍率下 进行测试, 循环 500 次后 $\mathrm{NCA} / \mathrm{AlF}_{3}$ 材料的容量保持 率为 $55.9 \%$, 远远高于原材料的 $11.7 \%$. 性能提升的 原因主要是以下 2 点: (1) 包覆物抑制了HF对电极材 料的侵蚀, 减少了过渡金属在电解液中的溶解, 从而 降低了电荷迁移电阻的增长; (2) 包覆物减小了材料 体积的变化, 从而阻止了材料在循环过程中的粉化. 原料和包覆后材料的 TEM图像及阻抗图如图4所示, 表明 $\mathrm{AlF}_{3}$ 包覆对保持材料形貌完整和抑制极化的显 著作用.

\section{4 锂化物}

不同于其他的包覆物, 锂化物作为 $\mathrm{Li}^{+}$的导体材 料, 能够更好地实现 $\mathrm{Li}^{+}$的嵌人和脱出, 提高材料的
循环性能和倍率性能; 同时, 某些锂化物本身就可以 作为电极材料, 在充放电过程中贡献容量, 从而减少 了包覆对于材料整体容量损失的影响. Liu等人 ${ }^{[28]}$ 通 过熔融盐法在NCA材料表面包覆 $3.0 \%$ (重量百分比) 的 $\mathrm{LiCoO}_{2}$. 在 $0.5 \mathrm{C}, 2.75 \sim 4.3 \mathrm{~V}$ 的测试条件下循环 50 次, $\mathrm{NCA} / \mathrm{LiCoO}_{2}$ 材料的首次放电比容量为 163.6 $\mathrm{mAh} / \mathrm{g}$, 容量保持率为 $95.8 \%$, 而未包覆材料的首次 放电比容量为 $154.3 \mathrm{mAh} / \mathrm{g}$, 容量保持率为 $87.9 \%$. 包 覆后材料的循环和倍率性能均有了一定提升. 电化 学阻抗测试结果表明在包覆层表面生成的 $\mathrm{NiO}$ 相的 减少是材料性能提升的主要原因.

Seino 等人 ${ }^{[29]} \mathrm{Li}_{4} \mathrm{Ti}_{5} \mathrm{O}_{12}$ 作为包覆物制得 NCA/ $\mathrm{Li}_{4} \mathrm{Ti}_{5} \mathrm{O}_{12}$ 材料, 在全固态电池中进行测试, 探究了不 同炦烧温度和包覆厚度对材料电化学性能的影响 ${ }^{[29]}$. 结果表明, 在 $300^{\circ} \mathrm{C}$ 煅烧、包覆厚度为 $5 \mathrm{~nm}$ 的材料具 有最佳性能. 过高的煅烧温度会导致Co扩散进人 $\mathrm{Li}_{4} \mathrm{Ti}_{5} \mathrm{O}_{12}$ 包覆层, 增强了包覆层内的电子传导, 使包 覆层不能通过抑制空间电荷层的扩展来降低电极的 阻抗. 而鉴于 $\mathrm{Li}_{4} \mathrm{Ti}_{5} \mathrm{O}_{12}$ 较差的电子传导性, 厚度过大 的包覆层也会对材料的充放电过程产生不利影响.
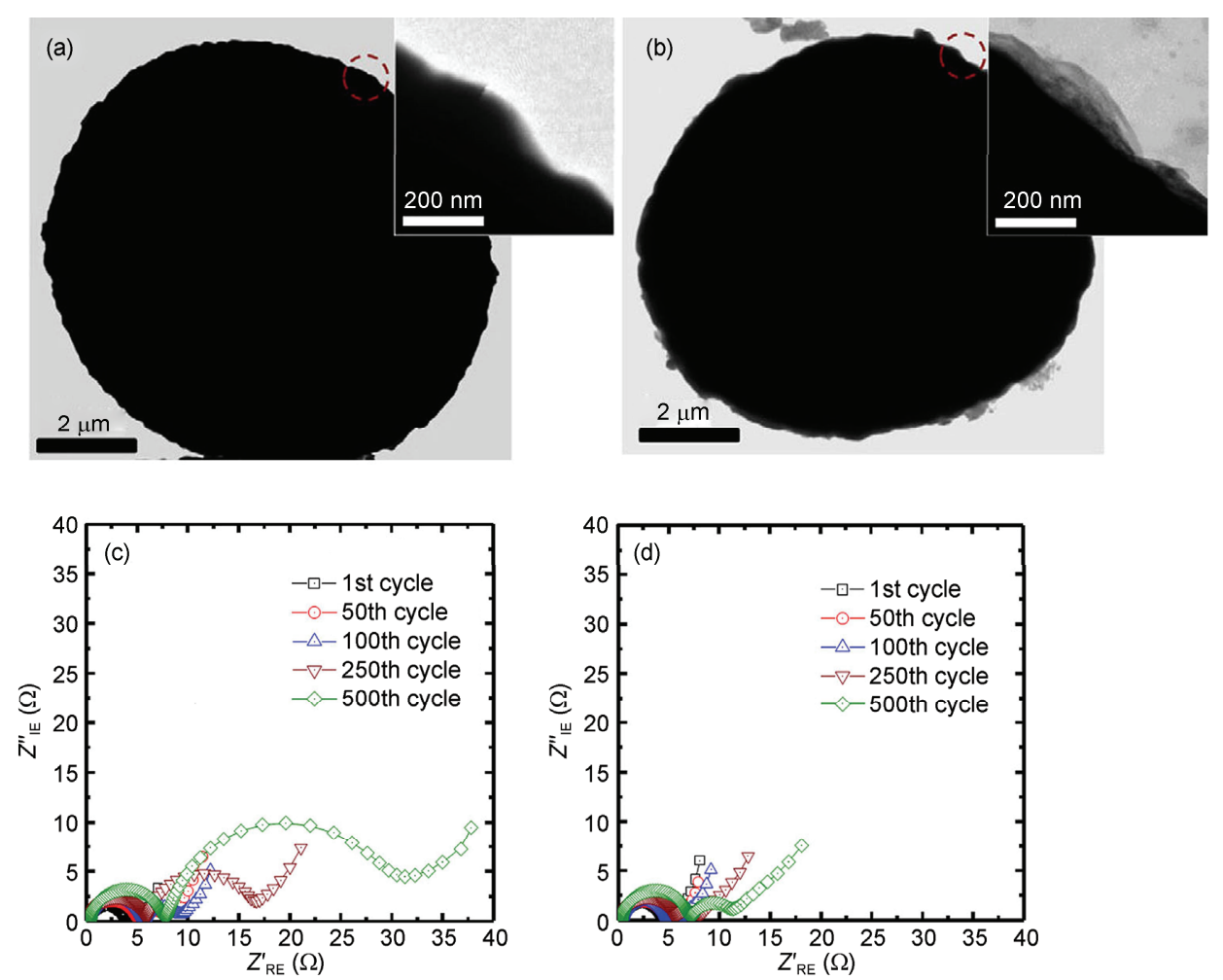

图 4 (网络版彩色) $\mathrm{NCA}(\mathrm{a})$ 和 $\mathrm{NCA} / \mathrm{AlF}_{3}(\mathrm{~b})$ 的 TEM图像. $\mathrm{NCA}(\mathrm{c})$ 和 $\mathrm{NCA} / \mathrm{AlF}_{3}(\mathrm{~d})$ 的阻抗图 ${ }^{[27]}$

Figure 4 (Color online) TEM images of NCA (a) and $\mathrm{NCA} / \mathrm{AlF}_{3}(\mathrm{~b})$. EIS plot of $\mathrm{NCA}(\mathrm{c})$ and $\mathrm{NCA} / \mathrm{AlF}_{3}(\mathrm{~d})^{[27]}$ 
$\mathrm{Ju}$ 和 $\mathrm{Ryu}^{[30]}$ 通过共沉淀法, 制备出核壳结构的 $\mathrm{Li}\left(\mathrm{Ni}_{0.8} \mathrm{Co}_{0.15} \mathrm{Al}_{0.05}\right)_{0.8}\left(\mathrm{Ni}_{0.5} \mathrm{Mn}_{0.5}\right)_{0.2} \mathrm{O}_{2}(\mathrm{NCANM})$ 材料, 内 核为 $\mathrm{LiNi}_{0.8} \mathrm{Co}_{0.15} \mathrm{Al}_{0.05} \mathrm{O}_{2}$, 外壳为 $\mathrm{LiNi}_{0.5} \mathrm{Mn}_{0.5} \mathrm{O}_{2}$. 通过 扫描电子显微镜结果可以看出, 包覆后材料外壳的 厚度大约为 $1.25 \mu \mathrm{m}$ (图5(a)). 在 2.8 4.3 V下按不同倍 率进行测试, 可以明显看出随着倍率的增大, 包覆后 材料的性能提升明显(图5(b)). 而在0.1 C下的循环测 试表明 NCANM材料较 NCA在容量保持上也有显著 提高(图 5(c)). $\mathrm{LiNi}_{0.5} \mathrm{Mn}_{0.5} \mathrm{O}_{2}$ 外壳阻止了电解液中的 HF对活性材料的侵蚀, 并限制了Co的溶解, 使材料 具有更好的倍率和循环性能. 同时, 差示扫描量热测 试表明材料的热稳定性也有了一定提高. 这是因为 具有较强热稳定性的 $\mathrm{LiNi}_{0.5} \mathrm{Mn}_{0.5} \mathrm{O}_{2}$ 外壳抑制了内核 材料晶格氧的释放, 从而提高了材料整体的热稳定 性. 同样通过共沉淀法, 胡国荣等人 ${ }^{[31]}$ 以三元材料 $\mathrm{LiNi}_{1 / 3} \mathrm{Co}_{1 / 3} \mathrm{Mn}_{1 / 3} \mathrm{O}_{2}$ 为包覆物, 通过高温炦烧合成了复 合层状材料 $\mathrm{Li}\left[\left(\mathrm{Ni}_{0.8} \mathrm{Co}_{0.15} \mathrm{Al}_{0.05}\right)_{0.97}\left(\mathrm{Ni}_{1 / 3} \mathrm{Co}_{1 / 3} \mathrm{Mn}_{1 / 3}\right)_{0.03}\right] \mathrm{O}_{2}$. 该复合材料具有核壳结构, $\mathrm{LiNi}_{1 / 3} \mathrm{Co}_{1 / 3} \mathrm{Mn}_{1 / 3} \mathrm{O}_{2}$ 包覆层 的厚度约为 $1.25 \mu \mathrm{m}$. 在 25 和 $55^{\circ} \mathrm{C}$ 条件下以 $0.2 \mathrm{C}$ 进行 测试, $25^{\circ} \mathrm{C}$ 循环 100 次, 未包覆样品的容量保持率为 $86.9 \%$, 而包覆后样品的容量保持率为 $96.2 \%$. 而在 $55^{\circ} \mathrm{C}$ 下, 包覆后样品循环 100 次的放电比容量为 163.2 $\mathrm{mAh} / \mathrm{g}$, 而未包覆样品在循环 50 次后的放电比容量只 有 $136.5 \mathrm{mAh} / \mathrm{g}$. 结果表明, 复合材料的循环性能, 尤其是高温下的循环性能有了很大提高.

\section{5 复合氧化物(锂化物-氧化物)}

关于将锂化物-氧化物作为复合氧化物对 NCA材 料进行包覆的方法同样有报道. Ito等人 ${ }^{[32]}$ 采用溶液 法, 配制甲醇锂和正丙醇锆的混合凝胶, 煅烧后在
$\mathrm{NCA}$ 材料上包覆 $\mathrm{Li}_{2} \mathrm{O}-\mathrm{ZrO}_{2}$ 复合氧化物, 并组装成全 固态电池进行测试. 对不同包覆量的样品的电荷转 移电阻进行测试, 结果表明 $0.5 \%$ (摩尔百分比)包覆量 电池的电荷转移电阻最低, 大大优于未包覆材料. 而 包覆后材料电池的整体电阻约为未包覆材料的 $1 / 4$, 电阻的降低促成了电化学性能的提升. 在 $1 \mathrm{~mA} / \mathrm{cm}^{2}$ 的高倍率下, $0.5 \%$ (摩尔百分比)包覆物的比容量约为 未包覆材料的 3 倍. 在 $25^{\circ} \mathrm{C}, 0.1 \mathrm{C}$ 条件下进行循环性 能测试, 100 次循环后包覆物和原料的容量保持率分 别为 $91.7 \%$ 和 $73.9 \%$; 同时包覆后样品在 $60^{\circ} \mathrm{C}$ 的较高 温度下测试也取得了较好性能.

以氢氧化锂和硼酸为原料, $\mathrm{Lim}$ 等人 ${ }^{[33]}$ 通过溶液 法制备出 $\mathrm{Li}_{2} \mathrm{O}-2 \mathrm{~B}_{2} \mathrm{O}_{3}(\mathrm{LBO})$ 包覆的 NCA/LBO 材料. $\mathrm{LBO}$ 包覆层的形成过程及 $\mathrm{Li}^{+}$在包覆层中的传输机理 如图6所示, 可以看出包覆层阻止了 HF对电极材料的 侵蚀, 并为 $\mathrm{Li}^{+}$提供了良好的扩散通道. 包覆后, 电 极材料的结构坞塌及过渡金属溶解受到抑制, 从而 提高了材料高温 $\left(55^{\circ} \mathrm{C}\right)$ 下的循环性能. $55^{\circ} \mathrm{C}$ 下以电流 为 $180 \mathrm{~mA} / \mathrm{g}$ 进行测试, 循环 100 次后包覆量为 $2 \%$ (重 量百分比)的 NCA/LBO材料的容量保持率为 $94.2 \%$, 远远高于未包覆材料的 $75.3 \%$. 因为具有较高的离子 传导能力, 包覆后材料的倍率性能也有了相应提高: 在 360 和 $900 \mathrm{~mA} / \mathrm{g}$ 的电流下, NCA/LBO材料的放电比 容量分别为 169 和 $147 \mathrm{mAh} / \mathrm{g}$, 而未包覆材料则对应 为 159 和 $133 \mathrm{mAh} / \mathrm{g}$. 另外, 由于包覆层抑制了电极材 料与电解液的副反应, 包覆后材料也展示了较好的 热稳定性：放热量为 $418.0 \mathrm{~J} / \mathrm{g}$, 少于原料的 $565.2 \mathrm{~J} / \mathrm{g}$. 从以上 2 个例子可以看出, 采用锂化物-氧化物作为 复合氧化物对 NCA材料进行包覆，可以很好地提高 电极材料的性能. 以此为基础, 尝试其他的氧化物组
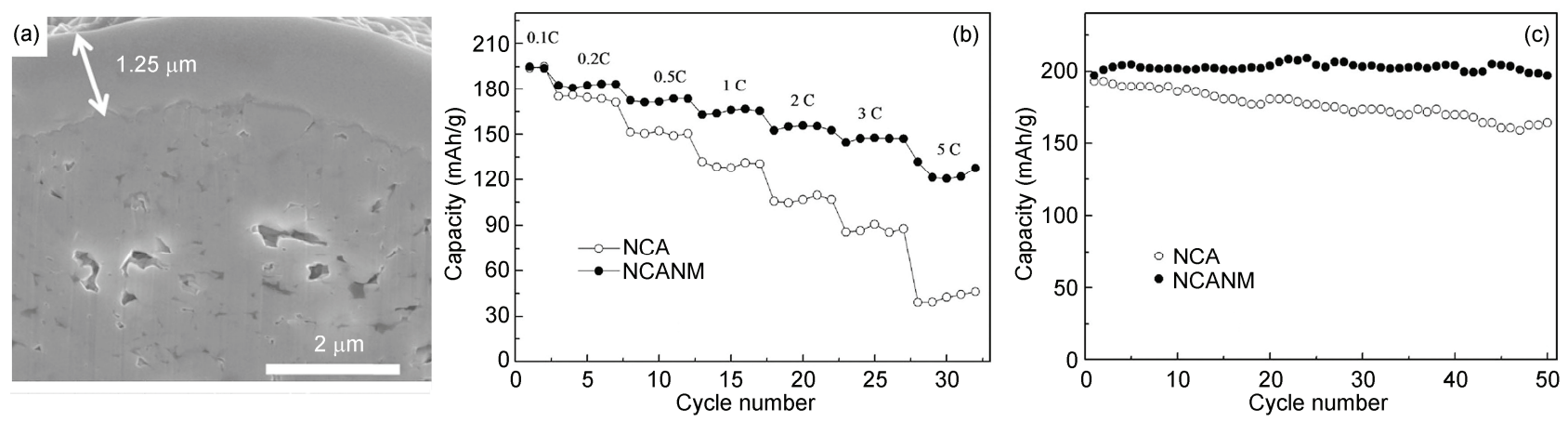

图 5 NCANM的剖面图(a), 倍率性能(b)和循环性能(c) ${ }^{[30]}$

Figure 5 Cross section image (a), discharge profiles at different rates (b) and cycling performances (c) of NCANM ${ }^{[30]}$ 


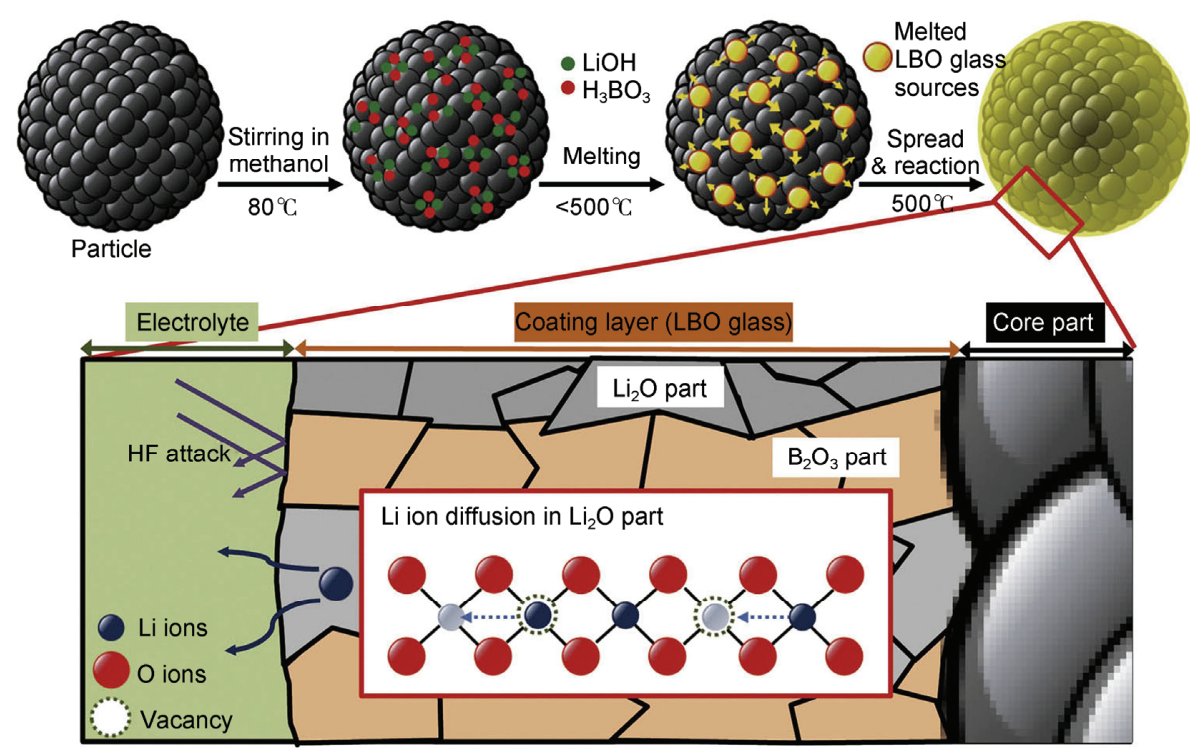

图 6 (网络版彩色)LBO包覆层形成过程及 $\mathrm{Li}^{+}$在包覆层中的传输机理 ${ }^{[33]}$

Figure 6 (Color online) Mechanism for preparation of coating layer and $\mathrm{Li}$ conducting in $\mathrm{LBO}\left(\mathrm{Li}_{2} \mathrm{O}-2 \mathrm{~B}_{2} \mathrm{O}_{3}\right)$ glass coating layer ${ }^{[33]}$

成, 或可成为未来的一个研究方向.

\section{6 磷酸盐}

磷酸盐作为正极材料的包覆物, 其对电极的包 覆作用原理与氧化物基本相同. 由于磷酸盐较氧化 物拥有更高的离子迁移能力和电子传递能力, 可以 更好地降低了电池阻抗, 从而对材料的倍率性能的 改善更加明显. $\mathrm{NCA}$ 与 $\mathrm{Ni}_{3}\left(\mathrm{PO}_{4}\right)_{2}$ 混合球磨后炦烧, 制 得 $\mathrm{NCA} / \mathrm{Ni}_{3}\left(\mathrm{PO}_{4}\right)_{2}$ 材料 ${ }^{[34]} . \mathrm{Ni}_{3}\left(\mathrm{PO}_{4}\right)_{2}$ 包覆层阻止了电 解液中的 HF对活性材料的侵蚀, 降低了电荷转移阻 抗, 从而提升了材料的循环性能. 在 $55^{\circ} \mathrm{C}, 0.5 \mathrm{C}$ 条件 下循环 100 次, 包覆后材料的容量保持率为 $75 \%$, 高 于未包覆材料的 53\%(图7(c)). 同时材料的倍率性能 也有一定提升. $10 \mathrm{C}$ 倍率下测试, $\mathrm{NCA} / \mathrm{Ni}_{3}\left(\mathrm{PO}_{4}\right)_{2}$ 与原 材料的放电比容量分别为 149 和 $127 \mathrm{~mA} / \mathrm{g}$. 对循环
100次之后的极片进行表征，结果发现包覆后材料的 表面基本保持原状，而原料表面则有明显被侵蚀的 痕迹(图7(a), (b)).

$\mathrm{NCA}$ 与 $\mathrm{FePO}_{4}$ 胶体混合, 抽滤后在 $80^{\circ} \mathrm{C}$ 烘干获得 $\mathrm{FePO}_{4}$ 包覆的 $\mathrm{NCA} / \mathrm{FePO}_{4}$ 材料 ${ }^{[35]}$. 与其他的包覆方法 相比, 该方法不需要高温煅烧或球磨, 整个实验过程 能耗较少, 非常温和. 对包覆后的材料进行表征, 可 以发现无定形的 $\mathrm{FePO}_{4}$ 均匀地包覆在材料表面. 包覆 后材料显示了良好的循环性能：以2C倍率进行测试, 循环 100 次后 $\mathrm{NCA} / \mathrm{FePO}_{4}$ 材料的容量保持率为 $88.3 \%$, 而未包覆材料只有 $70.7 \%$. 分别对循环 $1,50,100$ 次的 电池进行阻抗测试, 包覆后材料电池在循环过程中的 阻抗增长速度明显低于原料电池. 考虑到增加的阻抗 主要来源于电极材料与电解液的副反应, 表明无定形 的 $\mathrm{FePO}_{4}$ 包覆层能够抑制这些副反应, 生成更加稳固的
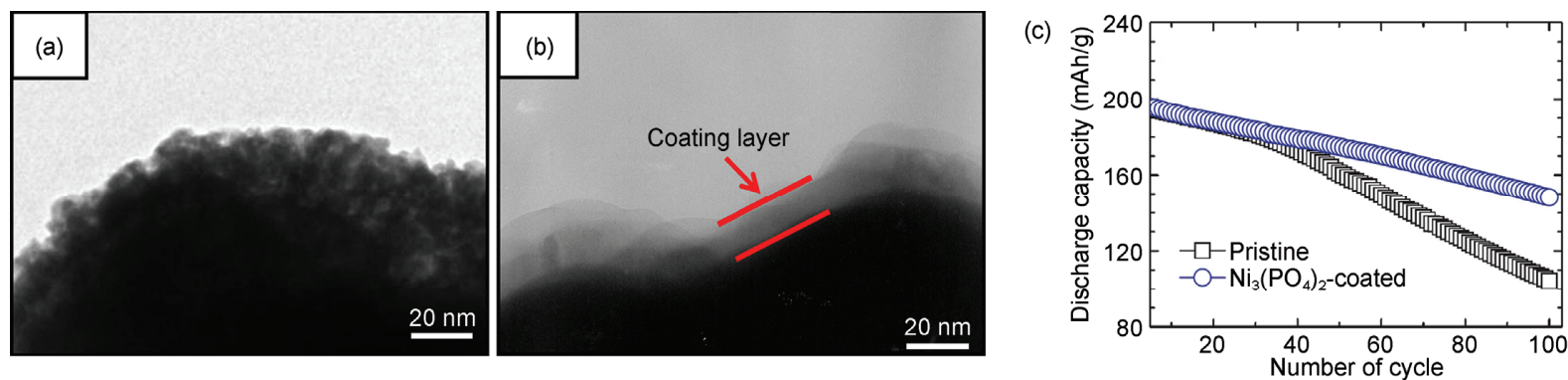

图 7 (网络版彩色) NCA(a)和 $\mathrm{NCA} / \mathrm{Ni}_{3}\left(\mathrm{PO}_{4}\right)_{2}$ (b)电极 $55^{\circ} \mathrm{C}$ 循环 100 次后的FE-TEM图像及循环性能对比(c) $)^{[34]}$

Figure 7 (Color online) FE-TEM images of NCA (a) and $\mathrm{NCA} / \mathrm{Ni}_{3}\left(\mathrm{PO}_{4}\right)_{2}$ (b) electrodes after 100 cycles at $55^{\circ} \mathrm{C}$. (c) Cycling performances ${ }^{[34]}$ 
固体电解质界面膜(SEI膜), 从而提高材料的循环性能.

\section{7 聚合物}

聚合物按照是否导电, 可以分为导电聚合物和 不导电聚合物. 其中导电聚合物因为具有特殊的电 学性质, 可以作为较好的电极材料包覆物. 采用原位 自稳定分散聚合的方法, Chung等人 ${ }^{[36]}$ 在NCA材料表 面包覆一层聚苯胺(Pani). 包覆后, 材料的循环和倍 率性能都有了一定提高. 由于聚苯胺包覆使材料的 结构稳定性大大增强, 所以在高倍率和高电压条件 下的性能提升效果更为明显. 值得注意的是, 相对于 大部分包覆物, 聚苯胺具有更好的电化学活性, 因此 聚苯胺包覆的电极材料较原材料具有更高的容量. 原料和包覆后材料的SEM图像及循环性能图如图 8所 示, 可以看出包覆后材料的放电容量略有上升, 循环 性能也得到一定提高.

\section{8 总结与展望}

作为新一代锂离子电池正极材料, $\mathrm{NCA}$ 材料由 于其较高的比容量、较低的成本, 有望取代目前的 $\mathrm{LiCoO}_{2}$ 材料与其他高比容量负极组装成新型大容量 电池 ${ }^{[37 ~ 39]}$, 具有广泛的应用前景. 为了进一步提升 材料的性能, 对 NCA材料进行包覆是较为有效的一 种手段. 表1总结了不同包覆物对NCA材料循环性能 的影响. 可以看出在包覆之后, 材料的循环性能都有 了一定提高. 不同的包覆物对材料的影响不尽相同. 其中氧化物/氟化物/磷酸盐包覆减少了电解液对材料 的腐蚀、抑制了材料与电解液的副反应, 但同时因为 其电化学惰性, 也可能对电子和 $\mathrm{Li}^{+}$的传导造成阻碍. 碳材料和复合氧化物则在保护材料本身的同时, 分 别提升了材料的导电性和 $\mathrm{Li}^{+}$传导能力. 而采用相对
稳定的某些电极材料(锂化物)作为包覆物, 与原料形 成核壳结构, 可以同时提升材料的导电性和 $\mathrm{Li}^{+}$的传 导能力, 并增强了材料的稳定性. 但这种结构同时也 存在着核壳之间的空隙在充放电过程中会逐渐扩大 的问题. 不同的包覆方法也会对 NCA材料的性能造 成影响. 目前常用的包覆方法主要分为物理包覆和 化学包覆. 其中物理包覆是将包覆物与原料混合后 进行机械球磨, 将包覆物包覆在原料上. 整个实验过 程较为简单, 缺点是能耗较大, 且包覆的均匀性有待 提高. 化学包覆则是在原料表面通过反应原位生成 包覆物, 其操作步骤通常较复杂, 但可以使包覆物在 原料表面包覆得更为均匀且结合更紧密. 一般材料 都可以采用这两种方法进行包覆, 实践中需要结合 原材料状态并根据实际需要来选择具体方法.

目前已经报道的对 NCA材料进行表面包覆的方 法都是较为通用的对锂电正极材料的包覆方法, 且 所采用的包覆材料也是较为传统的包覆材料. 考虑 到 NCA材料相对于其他正极材料的不同点(如表面残 留的碱性物质偏高、材料表面容易吸潮等), 可以针 对其特有性质, 设计出更加适合 NCA材料的包覆体 系, 考察包覆材料种类、包覆量、包覆方法对材料性 能的影响. 目前该方面的研究在其他富镍材料中已 有相关报道. 例如通过 $\mathrm{AlPO}_{4}$ 或 $\mathrm{Co}_{3}\left(\mathrm{PO}_{4}\right)_{2}$ 包覆来除去 富镍材料表面的残碱 $\left(\mathrm{LiOH}, \mathrm{Li}_{2} \mathrm{CO}_{3}\right)^{[40 \sim 42]}$, 以及通过 $\mathrm{NH}_{4} \mathrm{VO}_{3}$ 与残碱反应在 $\mathrm{LiNi}_{0.75} \mathrm{Co}_{0.11} \mathrm{Mn}_{0.14} \mathrm{O}_{2}$ 材料表面 形成 $\mathrm{V}_{2} \mathrm{O}_{5}-\mathrm{Li}_{x} \mathrm{~V}_{2} \mathrm{O}_{5}-\mathrm{LiNi}_{0.75-z} \mathrm{Co}_{0.11} \mathrm{Mn}_{0.14} \mathrm{~V}_{z} \mathrm{O}_{2}$ 的多重 壳结构 ${ }^{[43]}$. 考虑到 NCA材料与其他富镍材料具有相 似的性质, 可以尝试将以上包覆方法应用于NCA材 料. 同时，表面包覆虽然能够在一定程度上改善 NCA材料的电化学性能, 但其作用机理目前并不是 很明确, 与其他正极材料的包覆机理可能存在不同 (a)

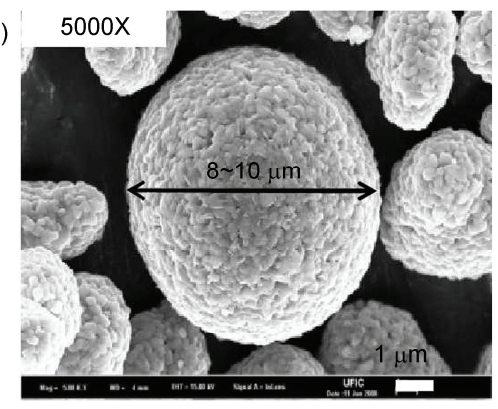

(b)

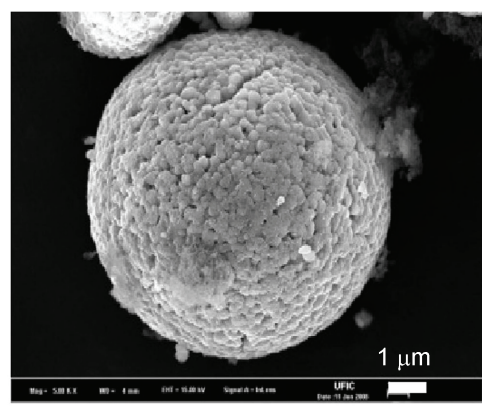

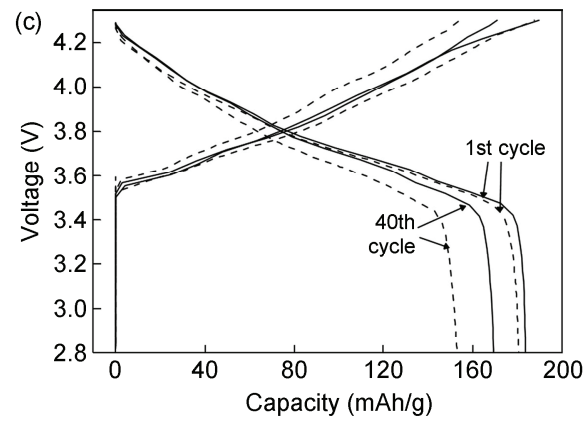

图 8 NCA (a) 和NCA/Pani (b)的SEM图像及循环性能对比(c) ${ }^{[36]}$

Figure 8 SEM images of NCA (a) and NCA/Pani (b). (c) Cycling performances ${ }^{[36]}$ 


\section{表 1 包覆物对NCA循环性能的影响}

Table 1 Effects of coating materials on the cycling performances of NCA

\begin{tabular}{|c|c|c|c|c|c|c|}
\hline 包覆物 & 测试温度 $\left({ }^{\circ} \mathrm{C}\right)$ & 倍率/电流 & 循环圈数 & 容量保持率 $(\%$, 包覆前) & 容量保持率(\%, 包覆后) & 文献 \\
\hline 碳材料 & - & $0.1 \mathrm{C}$ & 40 & 86 & 93 & [21] \\
\hline 石墨烯 & 25 & $55.6 \mathrm{~mA} / \mathrm{g}$ & 80 & 91 & 97 & {$[22]$} \\
\hline 纳米 $\mathrm{SiO}_{2}$ & 60 & $0.5 \mathrm{C}$ & 100 & 42 & 89 & {$[23]$} \\
\hline $\mathrm{CeO}_{2}$ & - & $0.5 \mathrm{C}$ & 100 & 78 & 86 & {$[24]$} \\
\hline $\mathrm{TiO}_{2}$ & 25 & $1.0 \mathrm{C}$ & 100 & 48 & 64 & {$[25]$} \\
\hline 纳米 $\mathrm{TiO}_{2}$ & 60 & $1.0 \mathrm{C}$ & 30 & 26 & 44 & {$[26]$} \\
\hline $\mathrm{AlF}_{3}$ & 55 & $1.0 \mathrm{C}$ & 500 & 12 & 56 & [27] \\
\hline $\mathrm{LiCoO}_{2}$ & - & $2.0 \mathrm{C}$ & 50 & 88 & 96 & {$[28]$} \\
\hline $\mathrm{LiNi}_{0.5} \mathrm{Mn}_{0.5} \mathrm{O}_{2}$ & - & $0.1 \mathrm{C}$ & 50 & 85 & 100 & {$[30]$} \\
\hline $\mathrm{LiNi}_{1 / 3} \mathrm{Co}_{1 / 3} \mathrm{Mn}_{1 / 3} \mathrm{O}_{2}$ & 25 & $0.2 \mathrm{C}$ & 100 & 87 & 96 & [31] \\
\hline $\mathrm{Li}_{2} \mathrm{O}-\mathrm{ZrO}_{2}$ & 25 & $0.1 \mathrm{C}$ & 100 & 74 & 92 & [32] \\
\hline $\mathrm{Li}_{2} \mathrm{O}-2 \mathrm{~B}_{2} \mathrm{O}_{3}$ & 55 & $180 \mathrm{~mA} / \mathrm{g}$ & 100 & 75 & 94 & [33] \\
\hline $\mathrm{Ni}_{3}\left(\mathrm{PO}_{4}\right)_{2}$ & 55 & $0.5 \mathrm{C}$ & 100 & 53 & 75 & [34] \\
\hline $\mathrm{FePO}_{4}$ & - & $2.0 \mathrm{C}$ & 100 & 71 & 88 & [35] \\
\hline 聚苯胺 & - & $0.1 \mathrm{C}$ & 40 & 85 & 92 & [36] \\
\hline
\end{tabular}

之处. 因此, 需要采用可靠的表征手段(例如电化学 与光谱原位联用技术), 对充放电过程以及循环前后 的NCA电极进行剖析, 研究电极组成结构形貌变化、 电极与电解液界面作用、电荷传输性质等, 进一步探 索包覆层的作用机制.

鉴于NCA材料有望成为未来广泛应用的锂离子 电池正极材料, 因此需重点关注材料的实用性. 对材 料进行改性的包覆方法也应遵循此思路进行设计, 包覆后NCA材料与电解液、负极材料的匹配性, 与电
池制作工艺的相容性、适应性等都需要加以考虑. 出 于规模生产的需要, 包覆过程的实验条件应尽可能 简单易行, 避免采用能耗较大的方法, 以节约成本. 此外，应尽量选用来源广泛、比较容易获得的包覆物, 有利于 NCA材料的大规模应用, 满足未来社会各领 域对电池材料的需求. 本文主要介绍了组成为 $\mathrm{LiNi}_{0.8}$ $\mathrm{Co}_{0.15} \mathrm{Al}_{0.05} \mathrm{O}_{2}$ 的 $\mathrm{NCA}$ 材料, 而更高 $\mathrm{Ni}$ 含量的 $\mathrm{NCA}$ 材料 因在容量和价格上更有优势目前也广受关注, 包覆 技术对于其改性具有更为重要的意义.

\section{参考文献}

1 Scrosati B, Garche J. Lithium batteries: Status, prospects and future. J Power Sources, 2010, 195: 2419-2430

2 Cheng F, Liang J, Tao Z, et al. Functional materials for rechargeable batteries. Adv Mater, 2011, 23: 1695-1715

3 Li H, Wang Z, Chen L, et al. Research on advanced materials for Li-ion batteries. Adv Mater, 2009, 21: 4593-4607

4 Xu B, Qian D, Wang Z, et al. Recent progress in cathode materials research for advanced lithium ion batteries. Mater Sci Eng, 2012, 73: 51-65

5 Wakihara M. Recent developments in lithium ion batteries. Mater Sci Eng, 2001, 33: 109-134

6 Xie J, Huang X, Zhu Z, et al. Hydrothermal synthesis of $\mathrm{LiNi}_{x} \mathrm{Co}_{1-x} \mathrm{O}_{2}$ cathode materials. Ceram Int, 2011, 37: 665-668

$7 \mathrm{Rim} \mathrm{H}$, Song J, Mumm D R. Electrochemical characteristics of $\mathrm{LiNi}_{0.7} \mathrm{Co}_{0.3} \mathrm{O}_{2}$ synthesized at $850^{\circ} \mathrm{C}$ from carbonates or oxides of $\mathrm{Li}$, Ni, and Co. Ceram Int, 2014, 40: 3511-3516

8 Gupta A, Chemelewski W D, Buddie Mullins C, et al. High-rate oxygen evolution reaction on Al-doped LiNiO 2 . Adv Mater, 2015, 27: 6063-6067

9 Liu Z, Zhen H, Kim Y, et al. Synthesis of $\mathrm{LiNiO}_{2}$ cathode materials with homogeneous Al doping at the atomic level. J Power Sources, 2011, 196: 10201-10206

10 Bie X, Gao Y, Yang X, et al. Observation of the second-order magnetic and reentrant spin-glass transitions in $\mathrm{LiNi}_{0.5} \mathrm{Mn}_{0.5} \mathrm{O}_{2} . \mathrm{J} \mathrm{Alloys}$ Compd, 2015, 626: 150-153 
11 Kan W H, Huq A, Manthiram A. Low-temperature synthesis, structural characterization, and electrochemistry of Ni-rich spinel-like $\mathrm{LiNi}_{2-y} \mathrm{Mn}_{y} \mathrm{O}_{4}(0.4 \leqslant y \leqslant 1)$. Chem Mater, 2015, 27: 7729-7733

12 Weaving J S, Coowar F, Teagle D A, et al. Development of high energy density Li-ion batteries based on $\mathrm{LiNi}_{1-x-y} \mathrm{Co}_{x} \mathrm{Al}_{y} \mathrm{O}_{2} . \mathrm{J}$ Power Sources, 2001, 97: 733-735

13 Cao H, Xia B, Xu N, et al. Structural and electrochemical characteristics of Co and Al co-doped lithium nickelate cathode materials for lithium-ion batteries. J Alloys Compd, 2004, 376: 282-286

14 Chen W, Li Y, Yang D, et al. Controlled synthesis of spherical hierarchical $\mathrm{LiNi}_{1-x-y} \mathrm{Co}_{x} \mathrm{Al}_{y} \mathrm{O}_{2}(0<x, y<0.2)$ via a novel cation exchange process as cathode materials for high-performance lithium batteries. Electrochim Acta, 2016, 190: 932-938

15 Ruan Z W, Gu H T, Shi H H, et al. Research progress of $\mathrm{LiNi}_{1-x-y} \mathrm{Co}_{x} \mathrm{Al}_{y} \mathrm{O}_{2}$ as cathode materials for lithium-ion battery (in Chinese). J Funct Mater, 2015, 1: 1007-1015 [阮泽文, 顾海涛, 史海浩, 等. 动力锂离子电池三元正极材料 $\mathrm{LiNi}_{1-x-y} \mathrm{Co}_{x} \mathrm{Al}_{y} \mathrm{O}_{2}$ 研究进展. 功能材 料, 2015, 1: 1007-1015]

16 Kondo H, Takeuchi Y, Sasaki T, et al. Effects of $\mathrm{Mg}$-substitution in $\mathrm{Li}(\mathrm{Ni}, \mathrm{Co}, \mathrm{Al}) \mathrm{O}_{2}$ positive electrode materials on the crystal structure and battery performance. J Power Sources, 2007, 174: 1131-1136

17 Woo S W, Myung S T, Bang H, et al. Improvement of electrochemical and thermal properties of $\mathrm{Li}\left[\mathrm{Ni}_{0.8} \mathrm{Co}_{0.1} \mathrm{Mn}_{0.1}\right] \mathrm{O}_{2}$ positive electrode materials by multiple metal (A1,Mg) substitution. Electrochim Acta, 2009, 54: 3851-3856

18 Lee K T, Jeong S, Cho J. Roles of surface chemistry on safety and electrochemistry in lithium ion batteries. Acc Chem Res, 2012, 46: $1161-1170$

19 Myung S T, Izumi K, Komaba S, et al. Role of alumina coating on Li-Ni-Co-Mn-O particles as positive electrode material for lithium-ion batteries. Chem Mater, 2005, 17: 3695-3704

20 Liu W, Oh P, Liu X, et al. Nickel-rich layered lithium transition-metal oxide for high-energy lithium-ion batteries. Angew Chem Int Ed, 2015, 54: 4440-4457

21 Chung Y M, Ryu S H, Ju J H, et al. A surfactant-based method for carbon coating of $\mathrm{LiNi}_{0.8} \mathrm{Co}_{0.15} \mathrm{Al}_{0.05} \mathrm{O}_{2}$ cathode in $\mathrm{Li}$ ion batteries. B Korean Chem Soc, 2010, 31: 2304-2308

22 Yoon S, Jung K N, Yeon S H, et al. Electrochemical properties of $\mathrm{LiNi}_{0.8} \mathrm{Co}_{0.15} \mathrm{Al}_{0.05} \mathrm{O}_{2}$-graphene composite as cathode materials for lithium-ion batteries. J Electroanal Chem, 2012, 683: 88-93

23 Cho Y, Cho J. Significant improvement of $\mathrm{LiNi}_{0.8} \mathrm{Co}_{0.15} \mathrm{Al}_{0.05} \mathrm{O}_{2}$ cathodes at $60^{\circ} \mathrm{C}$ by $\mathrm{SiO}_{2}$ dry coating for Li-ion batteries. $\mathrm{J}$ Electrochem Soc, 2010, 157: A625-A629

24 Xia S B, Zhang Y J, Dong P, et al. $\mathrm{CeO}_{2}$ surface modification to improve cycle and storage performance on lithium ion battery cathode material $\mathrm{LiNi}_{0.8} \mathrm{Co}_{0.15} \mathrm{Al}_{0.05} \mathrm{O}_{2}$ (in Chinese). Chin J Inorg Chem, 2014, 30: 529-535 [夏书标, 张英杰, 董鹏, 等. $\mathrm{CeO}_{2}$ 表面修饰提高锂 离子电池正极材料 $\mathrm{LiNi}_{0.8} \mathrm{Co}_{0.15} \mathrm{Al}_{0.05} \mathrm{O}_{2}$ 的循环及存储性能. 无机化学学报, 2014, 30: 529-535]

$25 \mathrm{Xu} \mathrm{Y,} \mathrm{Li} \mathrm{X,} \mathrm{Wang} \mathrm{Z,} \mathrm{et} \mathrm{al.} \mathrm{Structure} \mathrm{and} \mathrm{electrochemical} \mathrm{performance} \mathrm{of} \mathrm{TiO}_{2}$-coated $\mathrm{LiNi}_{0.8} \mathrm{Co}_{0.15} \mathrm{Al}_{0.05} \mathrm{O}_{2}$ cathode material. Mater Lett, 2015, 143: 151-154

26 Cho Y, Lee Y S, Park S A, et al. $\mathrm{LiNi}_{0.8} \mathrm{Co}_{0.15} \mathrm{Al}_{0.05} \mathrm{O}_{2}$ cathode materials prepared by $\mathrm{TiO}_{2}$ nanoparticle coatings on $\mathrm{Ni}_{0.8} \mathrm{Co}_{0.15} \mathrm{Al}_{0.05}(\mathrm{OH})_{2}$ precursors. Electrochim Acta, 2010, 56: 333-339

27 Lee S H, Yoon C S, Amine K, et al. Improvement of long-term cycling performance of $\mathrm{Li}\left[\mathrm{Ni}_{0.8} \mathrm{Co}_{0.15} \mathrm{Al}_{0.05}\right] \mathrm{O}_{2}$ by $\mathrm{AlF}_{3}$ coating. J Power Sources, 2013, 234: 201-207

28 Liu W, Hu G, Du K, et al. Synthesis and characterization of $\mathrm{LiCoO}_{2}$-coated $\mathrm{LiNi}_{0.8} \mathrm{Co}_{0.15} \mathrm{Al}_{0.05} \mathrm{O}_{2}$ cathode materials. Mater Lett, 2012, 83: $11-13$

29 Seino Y, Ota T, Takada K. High rate capabilities of all-solid-state lithium secondary batteries using $\mathrm{Li}_{4} \mathrm{Ti}_{5} \mathrm{O}_{12}-\mathrm{Coated} \mathrm{LiNi}_{0.8} \mathrm{Co}_{0.15}$ $\mathrm{Al}_{0.05} \mathrm{O}_{2}$ and a sulfide-based solid electrolyte. J Power Sources, 2011, 196: 6488-6492

$30 \mathrm{Ju} \mathrm{J} H$, Ryu K S. Synthesis and electrochemical performance of $\mathrm{Li}\left(\mathrm{Ni}_{0.8} \mathrm{Co}_{0.15} \mathrm{Al}_{0.05}\right)_{0.8}\left(\mathrm{Ni}_{0.5} \mathrm{Mn}_{0.5}\right)_{0.2} \mathrm{O}_{2}$ with core-shell structure as cathode material for Li-ion batteries. J Alloy Compd, 2011, 509: 7985-7992

31 Hu G R, Tan C P, Du K, et al. Performance of cathode material $\mathrm{LiNi}_{1 / 3} \mathrm{Co}_{1 / 3} \mathrm{Mn}_{1 / 3} \mathrm{O}_{2}$ coated $\mathrm{LiNi}_{0.8} \mathrm{Co}_{0.15} \mathrm{Al}_{0.05} \mathrm{O}_{2}$ (in $\mathrm{Chinese}$ ). Battery, 2013, (3): 143-146 [胡国荣, 谭潮溥, 杜柯, 等. 正极材料 $\mathrm{LiNi}_{1 / 3} \mathrm{Co}_{1 / 3} \mathrm{Mn}_{1 / 3} \mathrm{O}_{2}$ 包覆 $\mathrm{LiNi}_{0.8} \mathrm{Co}_{0.15} \mathrm{Al}_{0.05} \mathrm{O}_{2}$ 的性能. 电池, 2013, (3): 143-146]

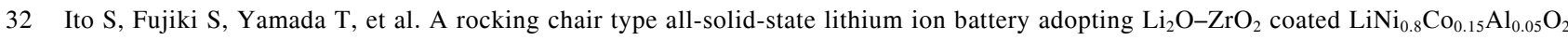
and a sulfide based electrolyte. J Power Sources, 2014, 248: 943-950

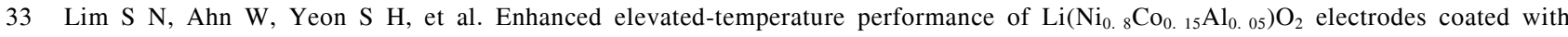
$\mathrm{Li}_{2} \mathrm{O}-2 \mathrm{~B}_{2} \mathrm{O}_{3}$ glass. Electrochim Acta, 2014, 136: 1-9

34 Lee D J, Scrosati B, Sun Y K. Ni $\mathrm{N}_{3}\left(\mathrm{PO}_{4}\right)_{2}$-coated $\mathrm{Li}\left[\mathrm{Ni}_{0.8} \mathrm{Co}_{0.15} \mathrm{Al}_{0.05}\right] \mathrm{O}_{2}$ lithium battery electrode with improved cycling performance at $55^{\circ} \mathrm{C}$. J Power Sources, 2011, 196: 7742-7746 
35 Huang B, Li X, Wang Z, et al. A facile process for coating amorphous $\mathrm{FePO}_{4}$ onto $\mathrm{LiNi}_{0.8} \mathrm{Co}_{0.15} \mathrm{Al}_{0.05} \mathrm{O}_{2}$ and the effects on its electrochemical properties. Mater Lett, 2014, 131: 210-213

36 Chung Y M, Ryu K S. Surface coating and electrochemical properties of $\mathrm{LiNi}_{0.8} \mathrm{Co}_{0.15} \mathrm{Al}_{0.05} \mathrm{O}_{2} /$ polyaniline composites as an electrode for Li-ion batteries. Bull Kor Chem Soc, 2009, 30: 1733-1737

37 Ortega-Vazquez M A. Optimal scheduling of electric vehicle charging and vehicle-to-grid services at household level including battery degradation and price uncertainty. IET Gener Transm Distrib, 2014, 8: 1007-1016

$38 \mathrm{Lu} \mathrm{L}$, Han X, Li J, et al. A review on the key issues for lithium-ion battery management in electric vehicles. J Power Sources, 2013, 226: 272-288

$39 \mathrm{Hu}$ X, Zhang K, Cong L, et al. Soil as an inexhaustible and high-performance anode material for Li-ion batteries. Chem Commun, 2015, 51: $15827-15830$

40 Cho J, Kim T J, Kim J, et al. Synthesis, thermal, and electrochemical properties of $\mathrm{AlPO}_{4}$-coated $\mathrm{LiNi}_{0.8} \mathrm{Co}_{0.1} \mathrm{Mn}_{0.1} \mathrm{O}_{2}$ cathode materials for a Li-Ion Cell. J Electrochem Soc, 2004, 151: A1899-A1904

$41 \mathrm{Hu}$ G R, Deng X R, Peng Z D, et al. Comparison of $\mathrm{AlPO}_{4}$-and $\mathrm{Co}_{3}\left(\mathrm{PO}_{4}\right)_{2}$-coated $\mathrm{LiNi}_{0.8} \mathrm{Co}_{0.2} \mathrm{O}_{2}$ cathode materials for Li-ion battery. Electrochim Acta, 2008, 53: 2567-2573

42 Zeng Y, He J. Surface structure investigation of $\mathrm{LiNi}_{0.8} \mathrm{Co}_{0.2} \mathrm{O}_{2}$ by $\mathrm{AlPO}_{4}$ coating and using functional electrolyte. J Power Sources, 2009, 189: 519-521

43 Park M H, Noh M, Lee S, et al. Flexible high-energy Li-ion batteries with fast-charging capability. Nano Lett, 2014, 14: 4083-4089 


\title{
Progress in surface coating on $\mathrm{LiNi}_{0.8} \mathrm{Co}_{0.15} \mathrm{Al}_{0.05} \mathrm{O}_{2}$ cathode materials for lithium-ion batteries
}

\author{
CONG Liang, LEI KaiXiang, WANG JiWei, WANG JianBin, MENG HuanJu, \\ CHENG FangYi \& CHEN Jun
}

Key Laboratory of Advanced Energy Materials Chemistry, Ministry of Education, College of Chemistry, Nankai University, Tianjin 300071, China

Cathode materials with high energy density and long life are currently the focus in the research and development of advanced lithium-ion batteries. Compared to traditional commercial layered $\mathrm{LiCoO}_{2}$, the nickel-rich cathode material with typical composition of $\mathrm{LiNi}_{0.8} \mathrm{Co}_{0.15} \mathrm{Al}_{0.05} \mathrm{O}_{2}$ (NCA) has attracted intensive interest because of advantages of low cost and high specific capacity (i.e., $\sim 200 \mathrm{mAh} / \mathrm{g}$ ). However, this promising cathode material suffers from problems, such as severe irreversible capacity loss and poor performance at high temperature due to the mixed cation distribution of $\mathrm{Li} / \mathrm{Ni}$ and the presence of high-valent $\mathrm{Ni}$ on charge that poses structural instability and strong oxidizing ability. There are generally two strategies to address the issues, namely, cation doping and the more extensively employed surface coating. In this article, we review recent progress made in surface coating of NCA cathode materials. Currently, the materials used for coating NCA mainly include carbonaceous materials (e.g., graphene), oxides and composite oxides (e.g., $\mathrm{SiO}_{2}, \mathrm{CeO}_{2}, \mathrm{TiO}_{2}$, and $\mathrm{Li}_{2} \mathrm{O}-2 \mathrm{~B}_{2} \mathrm{O}_{3}$ ), fluorides $\left(\mathrm{AlF}_{3}\right)$, lithium-containing compounds (e.g., $\mathrm{LiCoO}_{2}$ ), phosphates (e.g., $\mathrm{AlPO}_{4}$ and $\left.\mathrm{Co}_{3}\left(\mathrm{PO}_{4}\right)_{2}\right)$ and polymers (e.g., polyaniline). The surface-coated NCA shows better cycling performance as compared to the raw material. The effect of coating on electrode properties varies among different coated materials. The coating of oxides/fluorides/phosphates reduces the corrosion of electrolyte on the electrode and suppresses the side reaction between the electrolyte and the electrode, giving rise to better capacity retention. Carbonaceous materials and composite oxides exert similar protective effect and help to improve the electrical and ionic conductivity. Some stable lithium-containing compounds are coated on NCA to form a core-shell structure, facilitating both $\mathrm{Li}^{+}$insertion/delithiation and electron conduction with reduced electrode polarization and enhanced electrode stability. The coating methods also affect the electrode performance. Coating can be achieved through mechanical or chemical ways. The former is to mechanically mix the coating material and the raw NCA material by stirring or milling. Mechanical coating is simple in processing but is energy consuming and may incur structural/morphological break. Chemical coating is to generate in situ the coated material on the surface of raw material via chemical reactions. Complicated procedures are often required to make the coating layer uniformly distributed on target materials. As the coated materials are generally electrochemically inactive, their amount should be kept minimized to reduce expense of available capacity. Additionally, the type of coated materials and the thickness of coating layer should be optimized to make full advantage of coating. As a result of surface modification, the cyclability and thermal stability of NCA cathode materials can be greatly improved as compared to the pristine uncoated electrode.

Further research in surface coating of NCA is necessary if this attractive material is to find wide practical applications. First, in-depth understanding the surface properties of NCA and the complex NCA/electrolyte interface behaviors will enlighten the selection of coated materials. Current coating on NCA is almost totally based on previous knowledge of other lithium metal oxide electrodes. Ni-rich layered compounds may behave much differently, as exemplified by its particular sensitivity to water. Second, the underlined mechanism of the positive effect of surface coating on electrode performance remains elusive in many cases, which could resort to in situ analytical techniques that favor elucidation of structural evolution. Additionally, reproducibility and scalability surface coating process should be taken into consideration as NCA is viewed as a possible choice of cathode for electrical vehicles. The match of surface-coated NCA cathode with other battery components such as anode and electrolyte is also important point to be investigated. Other possible direction deserves to be mentioned is the combination of NCA with other active electrode materials, forming either a core-shell structure or a composite with gradient metal concentration. While herein we focus on $\mathrm{LiNi}_{0.8} \mathrm{Co}_{0.15} \mathrm{Al}_{0.05} \mathrm{O}_{2}$, there is increasing interest in NCA compounds with higher $\mathrm{Ni}$ content, which potentially renders higher energy density and lower material cost. Surface coating is expected to play even more important role in $\mathrm{LiNi}_{1-x-y} \mathrm{Co}_{x} \mathrm{Al}_{y} \mathrm{O}_{2}$ materials bearing a Ni:Li ratio near 1 .

lithium-ion battery, cathode materials, metal oxide, performance optimization, surface coating

doi: 10. 1360/N972016-00325 